\title{
QUALITY OF LIFE IN PATIENTS WITH DERMATOMYOSITIS AND POLYMYOSITIS IS CORRELATED WITH MUSCLE STRENGTH, FATIGUE AND DISABILITY
}

Cintia Maso de Sordi ${ }^{1, \star}$, Edgard Torres dos Reis Neto ${ }^{1}$, Samuel Katsuyuki Shinjo², Gerson Dierley Keppeke ${ }^{1}$, Emília Inoue Sato ${ }^{1}$

1.Universidade Federal de São Paulo, São Paulo (SP), Brazil. 2.Universidade de São Paulo São Paulo (SP), Brazil.

*Corresponding author: masocintia@gmail.com

\section{BACKGROUND}

Systemic autoimmune myopathies (SAMs) are characterized by chronic inflammation affecting skeletal muscle and causing mainly proximal muscular weakness. Quality of life in these patients has been poorly assessed and may have an important impact in the clinical outcome of these patients. The objective his study is to evaluate the quality of life in patients with dermatomyositis (DM) and polymyositis (PM) and its correlation with functional capacity, muscle weakness, muscle strength and fatigue.

\section{MATERIALS AND METHODS}

This is a cross-sectional study evaluating $34 \mathrm{DM}$ patients $16 \mathrm{PM}$ and 52 healthy controls matched by age and gender. Inclusion criteria: patients with DM or PM according to EULAR/ACR criteria; age over 18 years, informed consent form signature. Exclusion criteria: myopathies associated with other autoimmune diseases, neoplasm, statin and renal or cardiac insufficiency. Patients were evaluated according to the IMACS recommendation, including MMT-8, HAQ-M and VAS for disease activity. Quality of life was evaluated by Short-Form Health Survey-36 (SF-36) and fatigue by fatigue severity scale (FSS). Continuous variables were analyzed by T-student or Mann-Whitney tests. $P<0.05$ was considered significant.

\section{RESULTS}

Mean age of patients and controls were similar ( $50.9 \pm 14.0$ vs. $50.8 \pm 12.9 ; p=1.000), 39$ patients and 41 controls were female and the mean disease duration was $89.2 \pm 80.9$ months in patients group. Systemic autoimmune myopathies group presented worse quality of life in the domains of functional capacity (45.0 [5.0-100] vs. 85.0 [5.0-100]; $p \leq 0.001)$, physical capacity (75.0 [0.0-100] vs. 100 [0.0-100]; $p=0.030]$ general health (55.0 [5.0-92.0] vs. $77.0[10.0-100] ; p \leq 0.001)$ and vitality (50.0 [5.0-100] vs. 65.0 [0.0-100]; $p=0.010$ ); and presented more fatigue (4.9 \pm 1.6 vs. $3.2 \pm 1.5 ; p \leq 0.001)$, more disability (HAQ-M 0.63 [0.00-2.38] vs. 0.13 [0.00-1.75]; $p \leq 0.001$ ) and worse MMT-8 (70 [23-80] vs. 77 [60-80]; $p \leq 0.001$ ) when compared to controls. There was a positive correlation among MMT-8 and functional capacity (rho 0.526 ) and vitality (rho 0.519 ); a negative correlation between disability (HAQ-M) and functional capacity (rho -0.865), vitality (rho -0.530), physical aspects (rho -0.439), pain (rho -0.621), social aspects (rho -0.428) and emotional domains (rho -0.411); and a negative correlation between FSS and functional capacity (rho -0.639), vitality (rho -0.613), physical aspects (rho -0.491), pain (rho -0.573), social aspects (rho -0.569) and mental health (rho -0.506) domains of SF-36.

\section{CONCLUSION}

Systemic autoimmune myopathies patients have worse quality of life which is correlated with muscle strength, disability and fatigue. Strategies to improve quality of life in these patients must be included in consensus and guidelines. 